Artículo de investigación.

Cómo citar: M. Oble, J. Venegas, y A. Pérez "Evaluación del modelo hedónico en los huertos caseros de San Miguel Tlaixpan, Texcoco (México)", Inventum, vol. 14 , no. 27 , pp. 66-77. doi:10.26620/ uniminuto.inventum.14.27.2019.66-77

Editorial: Corporación Universitaria Minuto de Dios - UNIMINUTO.

ISSN: $1909-2520$

eISSN: $2590-8219$

Fecha de recibido: mayo 01 de 2019

Fecha de aprobado: junio 30 de 2019

Fecha de publicación: septiembre 01 de 2019

Conflicto de intereses: los autores han declarado que no existen intereses en competencia.
María Isabel Oble Delgadillo maria.oble@uimqroo.edu.mx Universidad Intercultural Maya de Quintana Roo, México

Alberto Pérez Fernández apfernandez@pampano.unacar.mx Universidad Autónoma del Carmen, México

José Apolonio Venegas Venegas Universidad Autónoma de Chiapas, México

\section{Evaluación del modelo hedónico en los huertos caseros de San Miguel Tlaixpan, Texcoco (México)}

\author{
Evaluation of the hedonic model in \\ the homes home of San Miguel Tlaixpan, \\ Texcoco (Mexico)
}

\author{
Avaliação do modelo hedônico nas \\ hortas de San Miguel Tlaixpan, \\ Texcoco (México)
}

\begin{abstract}
Resumen
En San Miguel Tlaixpan, Texcoco (México), se analizaron casas con y sin huerto, valoradas económicamente por servicios ambientales mediante precio hedónico de vivienda, más beneficio del atributo ambiental. Por ejemplo, una persona disfruta del huerto (ecosistema) para contemplar la fauna y la flora, para caminar y, por lo tanto, lo usaría para su bienestar social.

Con transformación Box-Cox en el proceso de estimación, el modelo es lineal. La función de precio hedónico explica los precios de viviendas en función de distintos atributos: tamaño del huerto tuvo un coeficiente de $\$ 20$, lo que indica que por cada hectárea del huerto, el precio aumenta en $\$ 20$ por m2; coeficiente de edad del huerto con un valor de $\$ 358$; si el material de construcción de las viviendas mejora que quiere decir dar mantenimiento y que cambie el material al de construcción con loza (bloques de cemento), mejora el material de pisos de cemento a piso de mármol entonces el huerto aumenta de precio en aproximadamente $\$ 8,332$ y si el huerto tiene disponibilidad de agua, el precio aumenta $\$ 6,740$. El modelo generado permite conocer la importancia de obtener propulsores del crecimiento económico y de la mejora en el bienestar humano de los huertos caseros para promover su conservación.
\end{abstract}

Palabras clave: vivienda, precio hedónico, huerto casero, estimación lineal, atributos ambientales.

\footnotetext{
Abstract

In San Miguel Tlaixpan, Texcoco, Mexico homes were analyzed with and without orchard, valued economically through environmental services through hedonic housing price plus profit environmental attribute. With the Box-Cox transformation in the estimation process, the model is linear. Hedonic price function of housing prices based on different attributes explained: size of orchard had a coefficient of $\$ 20$, indicating that for
} 
every hectare orchard, the price increases in $\$ 20$ by $\mathrm{m}^{2}$, the coefficient age of orchard with a value of $\$ 358$, if the construction material of the houses improves that means maintenance and that the material changes to the construction with earthenware (cement blocks), the material from cement floors to marble floor improves then the garden increases in price by approximately $\$ 8,332$ and if the home garden has water availability, $\$ 6,740$ price increases. The model generated allows knowing the economic, social and environmental importance of home gardens to promote their conservation.

Keywords: living place, hedonic price, home garden, envirmental atributs.

\section{Sumário}

Em San Miguel Tlaixpan, Texcoco (México), foram analisadas as casas com e sem pomar, valorizadas economicamente pelos serviços ambientais por meio de preços hedônicos da habitação, além do benefício do atributo ambiental. Por exemplo, uma pessoa desfruta do jardim (ecossistema) para contemplar a fauna e a flora, caminhar e, portanto, o usaria para seu bem-estar social.

Com a transformação Box - Cox no processo de estimativa, o modelo é linear. A função de preço hedônico explica os preços da habitação com base em diferentes atributos: o tamanho do jardim tinha um coeficiente de US $\$ 20$, o que indica que, para cada hectare do jardim, o preço aumenta em US $\$ 20$ por $\mathrm{m}^{2}$; coeficiente de idade do jardim com um valor de \$ 358; Se o material de construção das casas melhorar, isso significa manter e que o material muda para a construção com barro (blocos de cimento), o material do piso de cimento para o piso de mármore melhora, então o jardim aumenta o preço em aproximadamente $\$ 8.332$ e se o jardim tiver disponibilidade de água, o preço aumenta em US \$ 6.740. O modelo gerado nos permite conhecer a importância de obter fatores de crescimento econômico e melhoria do bem-estar humano das hortas domésticas para promover sua conservação.

Palavras-chave: habitação, preço hedônico, horta, estimativa linear, atributos ambientais. 


\section{INTRODUCCIÓN}

El huerto familiar es una de las tecnologías agroforestales en las que se presenta la asociación intensiva y multipropósito de cultivos para dar seguridad social y alimentaria [32]. Es un espacio habitual en las viviendas [25] que funciona como un agroecosistema con raíces tradicionales, donde los procesos de selección, domesticación, diversificación y conservación están orientados a la producción y reproducción de flora y fauna [26], [27], que evolucionan debido a la continua adecuación del espacio que ocupan [6], y generalmente, las especies vegetales son utilizadas como alimento [7], las plantas usadas para cada microhábitat de la huerta tienen un arreglo topológico que generalmente es en forma de hileras.

El arreglo espacial está relacionado con los estratos biológicos y las condiciones topográficas de la huerta [14]. Otra ventaja de los huertos es ofrecer una importante contribución al sistema social y ecológico [10] y de conservación de la diversidad biológica [44].

La existencia de huertos familiares está en estrecha relación con la preservación, las condiciones sociales, económicas y culturales de la familia, y el enriquecimiento, generación y apropiación de tecnología [26].

En América Latina el interés por los huertos caseros tradicionales ha crecido debido principalmente al auge alcanzado por los sistemas agroforestales [23].

El huerto casero como sistema agroforestal es una fuente primordial de sustento del resguardo indígena, ya que genera ingresos, alimentos, medicinas, combustible, materias primas para la artesanía [33], una fuente adicional de ingresos que requiere bajos insumos [45].

En gran medida, el interés por tal huerto se debe a que su estructura es parecida a la del bosque tropical: alta diversidad de especies en múltiples estratos vegetales, y el concepto de medio ambiente o ambiente natural se utiliza en ocasiones como sinónimo de hábitat o de ecosistema [35].

Los huertos familiares de San Miguel Tlaixpan se conforman de tres componentes espaciales: 1) la casa-habitación; 2) el patio jardín y 3) la huerta. Estos componentes difieren según los propósitos de cada familia, las modalidades de manejo y la división del trabajo por género, la composición florística y la abundancia de especies. Existen alrededor de 303 especies que orientan y estimulan la diversidad [14].

Los atributos ambientales pueden adicionar cantidades monetarias al valor total de los terrenos utilizados para el establecimiento de viviendas poblacionales [13].

La percepción de este valor se da en función de los servicios ambientales que ofrece, los cuales se pueden definir como el conjunto de condiciones y proceso naturales (incluyendo especies y genes) que la sociedad puede utilizar y que ofrecen las áreas naturales por su simple existencia [31].

Las preferencias de los individuos sobre espacios de atributos ambientales se pueden cuantificar de manera directa construyendo escenarios hipotéticos o indirectos a partir de comportamientos reales de los individuos en el mercado [16].

Los estudios reflejan que los efectos de las variables medioambientales son estadísticamente significativas, los jardines, espacios verdes y áreas con agua tienden a generar precios más altos [17].

El precio hedónico es un método indirecto que utiliza información de mercado con los costos evitados, que es utilizado para calcular el valor económico de bienes y servicios del ecosistema de calidad ambiental que afectan de manera directa a los precios de mercado de vivienda y los atributos ambientales relacionados con él, como el paisaje, aire limpio y belleza escénica [28].

Los atributos son expuestos a los agentes económicos de precios observados de productos diferenciados [39], y reflejan la relación entre el precio de un bien heterogéneo y las características que contiene [37].

La técnica de precios hedónicos es utilizada frecuentemente para medir la preferencia de la gente por tener una mejor calidad ambiental y que utilizan los mercados de trabajo y de bienes raíces como mecanismos de revelación de preferencias [41].

Este método se utiliza cuando existen características medioambientales que pueden tener efectos en los precios de bienes privados como terrenos o viviendas [16] y permite estimar los beneficios y los costos asociados con: la calidad ambiental 
(como la contaminación del aire y del agua, el ruido, etc.) y servicios ambientales estéticos (paisaje y recreación) [9]. Diversos estudios consideran el uso del método de precios hedónicos para analizar el mercado [2].

El precio observado de un producto puede ser analizado como la suma de los precios implícitos de los atributos [12]; aspectos como la existencia de escuelas de calidad influye en el precio de la vivienda [24], también escenarios culturales como la cercanía de edificios históricos [22]: Desde 2008, los diversos servicios ambientales y su tamaño tienen un impacto significativo en los precios de la vivienda [1].

Debido a los cambios que muestra la vida en la ciudad y el hacinamiento de la población en algunas áreas del Estado de México, la población ha optado por buscar colonias o ciudades que tengan viviendas con espacios verdes que brinden servicios estéticos y generen atributos como mejor calidad de aire y agua. La demanda de viviendas con estas características ha cambiado los precios de las mismas, por lo cual, es preciso conocer las características más valoradas por parte de la población al momento de comprar una vivienda con áreas verdes como un huerto casero.

Para este estudio se consideró un bien (vivienda), como un conjunto de características estructurales (superficie, número de habitaciones, número de baños), lugar donde esté localizada (distancia de la ciudad de Texcoco y distancia de las escuela) y adicionalmente de las características ambientales del entorno (calidad del aire, belleza escénica, agua rodada).

El principal objetivo fue evaluar los servicios ambientales como una externalidad o precio implícito de la vivienda en San Miguel Tlaixpan, Texcoco (México).

\section{MATERIALES Y MÉTODOS}

El estudio se realizó en San Miguel Tlaixpan, comunidad localizada en el cerro del Texcutzinco, Texcoco en el Estado de México a $9.5 \mathrm{~km}$ al este de la cabecera municipal y aproximadamente a $48 \mathrm{~km}$ de la Ciudad de México, dentro de las siguientes coordenadas geográficas; $19^{\circ} 30^{\prime} 00^{\prime \prime}$ a $19^{\circ} 31^{\prime} 00^{\prime \prime}$ latitud norte y $98^{\circ} 48^{\prime} 00^{\prime \prime}$ a $98^{\circ} 49^{\prime} 21^{\prime \prime}$ longitud oeste y con una altitud que varía entre 2,270 a $2,670 \mathrm{~m}$.
La metodología que se presenta en este trabajo consta de dos partes. La primera se caracteriza por la aplicación de 100 entrevistas de mayo a agosto de 2009 a casas con huertos caseros y sin huerto casero en la comunidad de San Miguel Tlaixpan, Texcoco.

La muestra se determinó mediante muestreo aleatorio simple, representativo al $95 \%$, con un error de estimación del $10 \%$, utilizando los datos del Censo de Población y Vivienda, del año 2006 de la Delegación de San Miguel Tlaixpan, existían aproximadamente 11,000 personas que corresponden a 1,500 familias.

La muestra es representativa para estimar la "disposición a pagar (DAA)", es decir, cómo los dueños de las casas perciben el servicio del huerto casero.

La fórmula que se utilizó para determinar la muestra es la siguiente:

$$
n=\frac{N Z^{2} p q}{N E^{2}+Z^{2} p}
$$

Dónde: $\mathrm{n}=$ tamaño de la muestra $=95 ; \mathrm{N}=$ tamaño de la población $=10,000 ; p=$ proporción de la población que esté dispuesto a pagar (0.5); q = proporción de la población que no esté dispuesto a pagar (0.5); $E$ = margen de error al $5 \%$ de la variable de interés; y $Z$ = nivel de confianza al $95 \%$.

La segunda parte consistió en aplicar un modelo de regresión por medio del método de precios hedónicos, cuyo objetivo es explicar las variables que determinan el precio de la vivienda y los precios implícitos de ellas, considerando principalmente si la casa habitación cuenta o no con la presencia de huerto casero.

\section{A. Modelo estimado}

Con el programa N-LOGIT 4.0 Box-Cox donde la variable dependiente es "precio" y las variables independientes son los atributos estructurales y ambientales, se procedió a elaborar una valoración económica-ecológica, este componente incluyó la medición de la disposición a pagar de los dueños de la vivienda con huerto casero, los precios implícitos de cada característica o atributo, con lo cual se obtiene la disposición marginal a 
pagar (DAPMg) por una unidad adicional de atributo, por lo que la DAP total no se puede evaluar. Así mismo, una vez conocido el precio del bien compuesto, es importante determinar el precio implícito adecuado que se debe asignar a las características que lo conforman [28].

\section{B. Precios hedónicos}

Esta técnica la desarrolló [38], y consiste en analizar mercados donde existen bienes heterogéneos, como la vivienda.

El análisis de precios hedónicos tiene un problema de endogeneidad [8], sin embargo, las ventajas del método hedónico son mayores que las desventajas [20].
Así, el precio $(\mathrm{P}(\mathrm{z}))$ de un predio está determinado por el valor de cada una de las particularidades del inmueble, atributos y externalidades. Así describe formalmente el equilibrio hedónico en función de los atributos externos, tal como lo expresa la ecuación 1:

$$
P(z)=f(z, \ldots, z)
$$

Se genera así la postura o disponibilidad a pagar (DAP) por los consumidores de vivienda e igualándola a la disponibilidad a aceptar (DAA) de los productores. Generalmente, la evidencia de la ecuación 1 o metodología de precios hedónicos, se realiza mediante transformación Box-Cox, expuesta a continuación [34].

\section{Transformaciones Box-Cox}

Tabla 1. Principales formas funcionales de los modelos hedónicos

\begin{tabular}{|l|l|l|}
\hline \multicolumn{1}{|l|}{ Forma Funcional } & Valor de Parámetros & Estimador \\
\hline Lineal & $\varnothing=\lambda=1 \mathrm{y} \beta \mathrm{ij}=0$ & MCO \\
\hline Semilog & $\varnothing=0, \lambda=1 \mathrm{y} \beta \mathrm{ij}=0$ & MCO \\
\hline Doble log & $\varnothing=\lambda=0$ y $\beta \mathrm{ij}=0$ & MCO \\
\hline Lineal Box - Cox & $\varnothing=\lambda=1 \mathrm{y} \beta \mathrm{ij}=0$ & MV \\
\hline Semilog Box - Cox & $\varnothing=0, \lambda=1 \mathrm{y} \beta \mathrm{ij}=0$ & MV \\
\hline Doble log Box - Cox & $\varnothing=\lambda=0 \mathrm{y} \beta \mathrm{ij}=0$ & MV \\
\hline
\end{tabular}

Nota: MCO: Mínimos Cuadrados Ordinarios, MV: Máxima Verosimilitud.

Fuente: [18].

La forma funcional Box-Cox cuadrática sin restricciones es la más empleada para estimar la función de precio hedónico (véase ecuación 2). Por otra parte, la ecuación 3 representa la función Box-Cox a estimar en la presente investigación. Con la cual, se pretende establecer la forma funcional más ajustada a la información disponible para capturar el cambio del valor monetario de los predios cercanos a una estación o portal de TransMilenio [34].

$$
\begin{aligned}
& P(Z)^{\theta}=\alpha_{0}+\sum_{i} \alpha_{i} z_{i}^{(\lambda)}+\sum_{j} \beta_{j} z_{j}^{(\lambda)}+ \\
& \sum_{i} \sum_{j} \beta_{i j} z_{i}^{(\lambda)} z_{j}^{(\lambda)} \\
& P(Z)^{\theta}=\beta_{0}+\sum_{i=1}^{k} \beta_{k} z_{k}^{(\lambda)}+e_{i} \\
& P(Z)^{\theta}=\frac{P(Z)^{\theta}-1}{\theta}, Z_{k}^{(\lambda)}=\frac{Z_{k}^{(\lambda)}-1}{\lambda}
\end{aligned}
$$


Donde $\theta$ y $\lambda$ son los parámetros de transformación y $e_{i}$ el término de perturbación (aleatorio).

Las variables independientes $z_{i}, z_{j}$ hasta $z_{k}$, representan cada uno de los atributos más relevantes de los predios y externalidades entre otros.

Los coeficientes $\beta 0, \beta 1, \beta 2, \ldots, \beta \mathrm{k}$ son las constantes del modelo; de acuerdo a su signo, representan la relación directa o inversa del atributo con el valor de la propiedad.

No obstante, según [18], dada la particularidad de complementariedad débil en los modelos de precios hedónicos, uno de los aspectos difíciles de tratar dentro de las transformaciones Box-Cox es el problema de autocorrelación espacial; dado que esta es una característica propia del modelo, deben incorporarse los principales aspectos de econometría espacial en las funciones Box-Cox [34].

$$
P(Z)^{\theta}=\alpha_{0}+\sum_{i} \alpha_{i} z_{i}^{(\lambda)}+\sum_{i} \sum_{j} \beta_{i j} z_{i}^{(\lambda)} z_{j}^{(\lambda)}
$$$$
Z^{(\lambda)}=\frac{Z^{\lambda}-1}{\lambda} \operatorname{Para} \lambda \neq 0 y Z^{(0)}=\operatorname{Ln}(Z)
$$

La estimación de la forma funcional Box-Cox para el modelo de precios hedónicos incluye las siguientes variables:

PRECIO: variable dependiente continua que representa el precio del predio por hectárea.

TAM: variable independiente continua que representa el tamaño de la vivienda con huerto casero y sin huerto en hectáreas.

EDA: variable independiente continua que representa la antigüedad de la vivienda.

1) MATCONT: variable independiente continua que representa el material con que está construida la vivienda.

2) ESCEN: variable independiente continua que representa la belleza escénica del huerto casero.

3) AIRE: variable independiente continua que representa el aire limpio que proporciona el huerto casero a la vivienda.
4) DISTC: variable independiente continua que representa la distancia en kilómetros de la vivienda a la ciudad de Texcoco.

5) AGUA: variable independiente dicotómica que toma el valor de 1, si la vivienda dispone de fuentes agua anual, y 0 , si no cuenta con esta.

Los argumentos o variables explicativas de la función están agrupados como atributos del entorno y se conforman de la siguiente expresión que describe la función hedónica. En ella, el precio de la vivienda $(P)$ es una función de sus características estructurales $(E)$ y de los atributos del entorno $(q)$, atributos estructurales: $P=P(E, q)[36]$. El modelo sobre formación de precios hedónicos señala que en el mercado se demandan y ofrecen productos con un conjunto [z] de atributos. La función de precios $\mathrm{p}[\mathrm{z}]$ se obtiene al igualar las cantidades demandadas $\mathrm{q}^{\mathrm{d}}[\mathrm{z}]$ con las cantidades ofrecidas $\mathrm{q}^{\mathrm{s}}$ [z] para todos los atributos zi $(i=1, . ., k)$ [21].

\section{La función de postura o \\ de regateo del consumidor}

Una implicación de la teoría del valor en economía es que los cambios en el nivel de satisfacción pueden expresarse en términos monetarios y esto posibilita la comparación entre distintas alternativas, lo que incrementa el bienestar del individuo, además de asumir algún grado de sustitución entre los bienes. Si todos los bienes pueden expresarse en términos monetarios, entonces se puede obtener la máxima cantidad de dinero que un individuo está dispuesto a pagar (DAP) por un incremento en la disponibilidad de algún bien, o la mínima cantidad de dinero que el individuo está dispuesto a aceptar (DAA) como compensación por renunciar voluntariamente a una mejora en su nivel de bienestar [43]. [11] y [4] encuentran que los valores de DAA son mayores a los de DAP. Esta sobreestimación en el valor del recurso se puede explicar por la presencia de un efecto ingreso subyacente en la pregunta de DAP. Adicionalmente, [19] sostiene que las diferencias encontradas en las estimaciones se deben a la presencia de un efecto sustitución.

Por esta razón, la conclusión lógica es que si no se desea sobredimensionar los beneficios agregados es aconsejable preguntar por DAP [46], [4].

Implícitamente, la función de postura (DAP), se puede expresar como: 


$$
\begin{aligned}
& D A P=\mathrm{f}(E, q, m, z ; u) \\
& U=u(E, q, z ; m-P) \\
& \frac{\partial \mathrm{DAP}}{\partial \mathrm{E}}=\frac{\partial \mathrm{f}(\mathrm{E}, \mathrm{q}, \mathrm{m}, \mathrm{z} ; \mathrm{u})}{\partial e_{i}}
\end{aligned}
$$

La DAA de los vendedores de un bien se expresa matemáticamente como:

Donde:

$$
D A A=f(E, q, k)
$$

$\mathrm{DAA}=$ es la disponibilidad $\mathrm{a}$ aceptar del productor $o$ vendedor.

$\mathrm{K}$ = es un parámetro que representa las características del productor (tecnología, capacidad financiera y de negociación, educación, etc.).

Cuando la DAP y la DAA son iguales, entonces la transacción ocurre; en consecuencia estas dos expresiones se vuelven equivalentes al precio $(P)$ del mercado.

$$
P=p(E, q, k) \text {. }
$$

La metodología de la investigación consiste en aplicar el método de precios hedónicos para la localidad de San Miguel Tlaixpan, Texcoco, para determinar qué atributos o características son importantes en la determinación de valor de las viviendas.

El modelo teórico antes descrito es la base para el desarrollo del trabajo econométrico que sirve para estimar, mediante una regresión, la función de precios hedónicos [42].

\section{RESULTADOS Y DISCUSIÓN}

La información analizada de un total de 100 encuestas -58 casas con huerto casero y 42 casas $\sin$ huerto- permitió identificar 87 propietarios originarios de la comunidad de San Miguel Tlaixpan y 13 de otras localidades de Texcoco y diferentes estados de la República mexicana. La información recopilada permitió determinar los precios implícitos de cada característica o atributo, con lo cual se obtiene la disposición marginal a pagar (DAPMg) por una unidad adicional de atributo.

\section{A. Estadísticas descriptivas (observaciones simples)}

Tabla 2. Modelo 1

\begin{tabular}{|l|r|r|r|r|r|}
\hline Variable & Coeficiente & Error estándar & b/St.Er & $\mid$ P $[\mathrm{Z} \mid>\mathrm{Z}] \mid$ & Media de X \\
\hline Edad & -0.2160 & 1.5380 & -0.1400 & 0.8890 & 54.3900 \\
\hline Área & 0.0480 & 0.1310 & 0.3650 & 0.7150 & 186.1200 \\
\hline No. Hab & -10.8510 & 15.7190 & -0.6900 & 0.4900 & 5.0600 \\
\hline No. Baños & 37.0740 & 49.5620 & 748.0000 & 0.4540 & 1.3850 \\
\hline MatCont & -28.6340 & 42.4550 & -0.6740 & 0.5000 & 2.1300 \\
\hline Antig & -0.7160 & 0.8710 & -0.8220 & 0.4110 & 54.2500 \\
\hline Agua & 61.5590 & 57.3850 & 1.0730 & 0.2830 & 0.4200 \\
\hline Escen & 95.5710 & 79.6260 & 1.2000 & 0.2300 & 0.8300 \\
\hline Aire & -32.3630 & 116.5030 & -0.2780 & 0.7810 & 0.9500 \\
\hline DisTe & 34.8550 & 55.1700 & 0.6320 & 0.5280 & 0.3030 \\
\hline DictC & 25.5990 & 31.3200 & 0.8170 & 0.4140 & 7.3150 \\
\hline Constante & -26.4840 & 222.6680 & -0.1190 & 0.9050 & \\
\hline Varianza y parámetros de transformación & 1.0000 & 0.0912 & 10.9620 & 0.0000 & \\
\hline Lambda & 42775.7930 & 50828.5720 & 0.8420 & 0.4000 & \\
\hline & & & & & \\
\hline
\end{tabular}

Fuente: elaboración propia. 
Tabla 3. Modelo 2

\begin{tabular}{|l|c|c|c|c|c|}
\hline Variable & Coeficiente & Error estándar & b/St.Er & $|\mathrm{P}[\mathrm{Z} \mid>\mathrm{Z}]|$ & Media de X \\
\hline Tam & 0.0015 & 0.0006 & 2.3560 & 0.0187 & 186.1200 \\
\hline Matcon & 0.0024 & 0.1510 & 0.0160 & 0.9871 & 2.1300 \\
\hline Antig & 0.0028 & 0.0028 & 1.0010 & 0.3167 & 54.2500 \\
\hline Agua & 0.4432 & 0.2214 & 2.0020 & 0.0453 & 0.4200 \\
\hline ESCen & 0.5812 & 0.3059 & 1.9000 & 0.0574 & 0.8300 \\
\hline Aire & 0.2637 & 0.4765 & 0.5540 & 0.5799 & 0.9500 \\
\hline DISTC & 0.1358 & 0.1220 & 1.1130 & 0.2659 & 7.3150 \\
\hline Constante & 2.1541 & 0.9075 & 2.3740 & 0.0176 & \\
\hline Varianza y parámetros de transformación & & & \\
\hline Lambda & 0.0000 & 0.0642 & 0.0000 & 1.0000 & \\
\hline Sigma-sq & 0.7688 & 0.4291 & 1.7920 & 0.0732 & \\
\hline
\end{tabular}

Fuente: elaboración propia.

Tabla 4. Modelo 3

\begin{tabular}{|l|c|c|c|c|c|}
\hline \multicolumn{1}{|c|}{ Variable } & Coeficiente & Error estándar & $\mathrm{b} / \mathrm{St}$.Er & $|\mathrm{P}[\mathrm{Z} \mid>\mathrm{Z}]|$ & Media de $\mathrm{X}$ \\
\hline Tam & 0.0020 & 0.0007 & 2.8900 & 0.0030 & 186.1200 \\
\hline Edad & 0.0358 & 0.0065 & 5.5000 & 0.0000 & 54.3900 \\
\hline Agua & 0.6740 & 0.2630 & 2.5600 & 0.0104 & 0.4200 \\
\hline MatCon & 0.8332 & 0.1531 & 5.4400 & 0.2630 & 2.1300 \\
\hline Varianza y parámetros de transformación & & & \\
\hline Lambda & 0.0000 & 0.0492 & 0.0000 & 1.0000 & \\
\hline Sigma-sq & 1.3011 & 0.4841 & 2.6870 & 0.0072 & \\
\hline
\end{tabular}

Fuente: elaboración propia.

\section{B) Análisis de los modelos estimados}

En el modelo 1 (tabla2) y modelo 2 (tabla3) había variables no significativas (utilizando un $\alpha=0.01$ ), por lo que se procedió a descartar variables, con lo que quedó el modelo 3 (tabla 4) como el mejor. Los coeficientes para el análisis se obtuvieron multiplicando por 10,000, debido a que en la estimación del modelo la variable precio fue dividido por 10,000 a fin de reducir su escala. Con la transformación Box-Cox planteada en el proceso de estimación se encontró el $\lambda$ que maximiza la función de verosimilitud igual a 1, por lo tanto, en el modelo 3 (tabla 4) se está suponiendo que las variables explicativas del precio de los huertos son lineales. La prueba de la razón de verosimilitud para saber si lambda es restringida y estadísticamente significativa se representa de la siguiente forma: 


\section{$\mathrm{H}_{0}$ (No hay significancia): $\lambda=0$ \\ $\mathrm{H}_{1}$ (Si hay significancia) $: \lambda \neq 0$}

Evaluando $\lambda$ y $r$ (restringido $)=0$ en el logaritmo de la función de verosimilitud, se tiene que:

$$
\operatorname{LnL}(R)=-679.0996
$$

Evaluando $\lambda$ y $M V$ (no restringido $)=1$, es decir:

$$
\operatorname{LnL}(N R)=-155.0525
$$

El estadístico está dado por:

$$
\begin{gathered}
-2[\operatorname{Ln} L(R)-\operatorname{LnL}(N R)]= \\
-2[-679.0996+155.0525]=1,048.0942
\end{gathered}
$$

El valor crítico para una distribución Chi-cuadrada ( 1 grado de libertad) es de 2.71; Como 1,048 es mayor que 2.71 , se rechazó la hipótesis nula y se optó por la hipótesis alterna, aceptando que la forma funcional de la regresión es lineal.

\section{C) Características estructurales que explican el precio de los huertos caseros}

La variable tamaño del huerto (TAM) con un valor del coeficiente de 20.09, muestra que por cada hectárea del huerto, el precio aumenta en aproximadamente $\$ 20 \mathrm{~m}^{2}$. El coeficiente de esta variable es positivo, lo que indica una relación directa entre el tamaño del huerto y el precio por hectárea.

La variable edad del huerto (EDA), también mostró un signo positivo, lo que demuestra la existencia de una relación directa entre la edad y el precio por hectárea de tierra, el valor del coeficiente de 358.17 indica que, por cada incremento en un año en la edad del huerto, la hectárea se valorizara en $\$ 358$.

El material de construcción (MATCON) fue otra variable con importancia en el modelo, muestra que a medida que mejora el material de construcción de las viviendas al interior del huerto, el huerto aumenta de precio en aproximadamente $\$ 8,332$. El signo encontrado para esta variable fue positivo.

El único atributo ambiental significativo en la explicación del precio de los huertos caseros resultó ser la disponibilidad de agua, el cual fue capturado como una variable cualitativa. La variable disponibilidad de agua tuvo un coeficiente igual a 0.6740; es decir, que la diferencia en precio entre dos huertos con características idénticas, pero donde uno de ellos dispone de agua y el otro no es de $\$ 6,740$. El signo del coeficiente es positivo, indica que el atributo disponibilidad de agua es una variable que influye positivamente en el valor del huerto casero.

El precio del suelo es una de las principales variables que explican el precio de la vivienda [39]. Para este estudio se consideró el aspecto ambiental como un enfoque para explicar los precios de la vivienda, al igual que [30] se enfatiza la valoración económica de los bienes en función del patrimonio natural. [15] analizaron la venta de un millón de casas en Inglaterra entre 1996 y 2008 y concluyeron que el mercado de la vivienda revela un carácter de utilidad sustancial en las viviendas con jardines privados y servicios ambientales.

Los modelos hidroeconómicos (MHE) permiten analizar los problemas de gestión del agua considerando de forma explícita las interacciones entre ingeniería, economía y medio ambiente, esto proporciona resultados más relevantes para la toma de decisiones en un entorno tan complejo como el del agua. La interacción entre los sistemas naturales y humanos ha llevado a hablar de sistemas acoplados hombre-naturaleza.

En este sentido, una línea de trabajo novedosa es la integración de modelos hidroeconómicos con modelos de simulación de agente [48].

La existencia de áreas con agua puede incrementar la renta de una casa hasta el 57 \% [1].

La investigación realizada por [5], en donde demuestran que la contaminación del sector influye significativamente en los precios de las viviendas, al igual que el agua. Los inmuebles más alejados del parque central tienen un precio de alquiler menor que los que se encuentran próximos; es así que, por cada $100 \mathrm{~m}$ de distancia de la vivienda al centro, el precio de alquiler disminuye en promedio un $5 \%$. 
Esta relación negativa se observa, así mismo, en el trabajo realizado por [29], donde por cada metro que la vivienda se aleja de una zona con áreas verdes, su precio disminuye en 3.83 euros. [40] encontraron que un $97 \%$ de las viviendas aumentaron de precio si se encontraban cerca de árboles y áreas verdes. [15], analizaron la venta de un millón de casas en Inglaterra entre 1996 y 2008 y concluyeron que el mercado de la vivienda revela un carácter de utilidad sustancial en las viviendas con jardines privados y servicios ambientales.

[3] demuestran que esta variable también afecta a los precios de los depósitos industriales en Holanda, donde porcada kilómetro que la propiedad se aleje del parque central el precio disminuye un $10 \%$ [47].

En estudios futuros se podría incorporar información de variables ambientales adicionales, como nivel de ruido, grado de contaminación del agua, número de árboles por sector, entre otras.

Los gobiernos seccionales, conjuntamente con el Gobierno nacional, deberían generar estadísticas ambientales detalladas por ciudad y, de esta manera, permitir la cuantificación correcta del valor económico de aquellos bienes que no poseen mercado, es decir, de los bienes ambientales [47].

\section{CONCLUSIONES}

El modelo hedónico estimó un precio final adecuado que refleja la valía real de una vivienda, atendiendo las características implícitas que esta posee y demuestró la importancia y la significancia individual de las características propias de una vivienda en los precios de venta, por contener huerto casero. Se expuso un método que permitió valorar una vivienda con huerto casero y sin huerto, no obstante, y a la vista de los resultados obtenidos, la eficacia del método presenta una gran dependencia de dos factores. En primer lugar, de que la variable ambiental explique un porcentaje significativo del valor de mercado de las viviendas, y en segundo lugar, de la transparencia del mercado.

El método de los precios hedónicos ha permitido descubrir todos los atributos de las viviendas que explican su precio, discriminando el componente cuantitativo de estos, asignando a cada una de las características cualitativas del bien un precio implícito. Este precio estaría reflejado por la disposición marginal a pagar de la persona por una unidad adicional del atributo.
Al adquirir una vivienda con huerto casero, no solo se están comprando unas hectáreas de tierra, sino que también se está seleccionando un entorno que tiene una serie de características (disponibilidad de agua, distancia de la vivienda hacia el centro de la ciudad de Texcoco o a las escuelas), como también a la elección de determinadas características de tipo ambiental que las personas están dispuestas a pagar por ellas. El diferencial de precio entre dos viviendas muy similares en sus características, excepto en la disponibilidad de agua, es $\$ 6,740$ que refleja el valor de ese atributo que, en principio, carece de un precio explícito en el mercado.

\section{REFERENCIAS}

[1] A. Baranzini y C. Schaerer, "A sight for sore eyes: Assessing the value of view and land use in the housing market", J Hous Econ, vol. 3, n. ${ }^{\circ}$ 20, pp. 191-199, 2011.

[2] K. Bartholomew y R. Ewing, "Hedonic Price effects of pedestrian -and transit- oriented development". J. Plan. Lit, vol. 1, n. ${ }^{\circ}$ 26, pp. 18-34, 2011.

[3] J. Beekmans, P. Beckers, E. V. Krabben, y K. Martens, "A hedonic price analysis of the value of industrial sites". Journal of Property Research, vol. 31, n. ${ }^{\circ}$ 2, pp. 108-130, 2014.

[4] C. R. Bishop y A. T. Heberlein, "Measuring values of extra market goods: Are Indirect Measure biased?", Am. J. Agric. Econ. vol. 61, n. ${ }^{\circ}$ 5, pp. 926-930, 1979.

[5] D. Brasington y D. Hite, "Demand for environmental quality: a spatial hedonic analysis". Regional Science and Urban Economics, vol. 1, n. ${ }^{\circ}$ 35, pp. 57-82, 2005.

[6] M. Cano-Ramírez, B. De la Tejera, A. Casas, L. Salazar y R. García-Barrios, "Migración rural y huertos familiares en una comunidad indígena del centro de México", Botanical Sciences, vol. 3, n. ${ }^{\circ}$ 90, pp. 287-304, 2012,

[7] R. Chablé-Pascual, D. J. Palma-López, C. J. Vázquez-Navarrete, O. Ruíz-Rosado, R. MariacaMéndez y J. Ascencio-Rivera, "Estructura, diversidad y uso de las especies en huertos de la Chontalpa, Tabasco, México". Ecosistemas y Recursos Agropecuarios. vol. 4, n. ${ }^{\circ}$ 2, pp. 23-39, 2015. 
[8] M. Costanigro y J. J. Mc Cluskey, "Hedonic Price analysis in food market". En J. L. Lusk, J. Roosen, y J. F. Shogren, eds., The Oxford Handbook of The Economics of Food Consumption and Policy, Reino Unido: Oxford University Press, 2013, 902 p.

[9] E. Cristeche y J. A. Penna, Métodos de valoración económica de los servicios ambientales, Buenos Aires: Instituto Nacional de Tecnología Agropecuaria (INTA), 2008.

[10] B. Degenhart, "La agricultura urbana: un fenómeno global”, Nueva Sociedad, n. ${ }^{\circ} 262$, pp. 133-146, 2016.

[11] P. A. Diamond y J. A. Hausman, "Contingent Valuation: is Some Number Better than No Number?", J. Econ. Perspect, vol. 8, n. ${ }^{\circ} 4$, pp. 45-64, 1994.

[12] O. M. J. Estrella, E. Defrancesco y A. Gennari, "Thewinehedonic price models in the "Old and New World': state of the art", Revista de la Facultad de Ciencias Agrarias. vol. 1, n. ${ }^{\circ}$ 44, pp. 205-220, 2012.

[13] E. M. Fernandes, B. A. S. González, Y. B. Cañete, J. M. C. González, y M. V. González, "Determinantes del precio del terreno para vivienda en Pedro Juan Caballero: una estimación hedónica. Brasil". Anais do Encontro Científico de Administração, Economia e Contabilidade, vol. 1, n. ${ }^{\circ} 1$, pp. 12-25, 2015.

[14] Á. C. Gaytán, H. Vibrans, G. H. Navarro y V. M. Jiménez, "Manejo de Huertos Familiares Periurbanos de San Miguel Tlaixpan, Texcoco, Estado de México", Bol. Soc. Bol, vol. 69, pp. 39-62, 2001.

[15] S. Gibbons, S. Mourato y G. M Resende. value of English nature: A hedonic price approach", Environmental \& Resource Economics. vol. 2, n. ${ }^{\circ}$ 57, pp. 175-196 2014.

[16] G. M González, "Preferencias de los individuos por los espacios recreativos: dos aplicaciones en Galicia". Estudios de Economía Aplicada, vol. 16, n. ${ }^{\circ}$ 3, pp. 93-110, 2000.

[17] P. Gottlieb, Hedonic Models: Valuation of Urban Parks, tesis, Departamento de Economía Agrícola y de Recursos. Universidad de Maryland, College Park, 1996.

[18] C. T Haab y E. K. McConnell, Valuing Environmental and Natural Resources: The econometrics of non-market valuation, Gran Bretaña: MPG Books Ltd. 2002.

[19] M. Hanemann, J. Loomis y B. Kanninen, "Statistical Efficiency of Double-bounded Dichotomous Choice Contingent Valuationll", Am. J. Agric. Econ., vol. 73, n. ${ }^{\circ}$ 4, pp. 12551263, 1991.

[20] R. J. Hill, "Hedonicprice indexes forresidentialhousing: A survey, evaluation and taxonomy", Journal of Economic Survey, vol. 27, n. ${ }^{\circ}$ 5, pp. 879-914, 2013.

[21] M. Jansson y A. Axel, "Función de precios hedónicos de viviendas y adaptación del Test Reset en modelos no lineales. Aplicación del modelo Box \& Cox a los precios de las viviendas de la ciudad de Catamarca", Pharos. vol. 7, n. ${ }^{\circ}$ 2, pp. 42-59, 2000.

[22] F. Lazrak, P. Nijkamp, P. Rietveld y J. Rouwendal, The market value of cultural heritage in urban areas: an application of spatial hedonic pricing, Journal of Geographical Systems. vol. 1, n. ${ }^{\circ}$ 16, pp. 89-114, 2014.

[23] R. Lok, Introducción a los huertos caseros tradicionales tropicales, Turrialba, Costa Rica: CATIE, 1998.

[24] S. Machin, "Houses and school: valuation of school quality through the housingmarket", Labour Economics., vol. 6, n. ${ }^{\circ} 18$, pp. 723729, 2011.

[25] M. G. I. Manzanero, M. A. Flores y E. S. Hunn, "Los huertos familiares zapotecos de San Miguel Talea de Castro, Sierra Norte de Oaxaca, México", Etnobiología. vol. 1, n. ${ }^{\circ}$ 7, pp. 9-29, 2009.

[26] M. R. Mariaca, J. A. González y T. M. Lerner, "El huerto familiar en México: avances y propuestas", en: O. J. F. López, G. A. Aragón y R. A. M. Tapia, eds., Avances en Agroecología y Ambiente, México: Benemérita Universidad Autónoma de Puebla. . 2007. pp. 119-138.

[27] M. R. Mariaca, El huerto familiar del sureste de méxico. secretaría de recursos naturales y protección ambiental del estado de Tabasco, México: El Colegio de la Frontera Sur. 2012.

[28] L. J. C. Mendieta, Manual de valoración económica de bienes no mercadeables, 2.a ed., Bogotá: Universidad de los Andes, 2005. 
[29] A. B. Morancho, "A hedonic valuation of urban green areas", Landscape and urban planning, vol, 66, n. ${ }^{\circ}$ 1, pp. 35-41, 2003.

[30] G. Z. I. Novoa, "Valoración económica del patrimonio natural: las áreas naturales protegidas”, Espacio y Desarrollo, n. ${ }^{\circ} 23$, pp. 131154, 2011.

[31] Organización de las Naciones Unidas para la Agricultura y la Alimentación (FAO). Estado mundial de la agricultura y la alimentación: Pagos a los agricultores por servicios ambientales, Roma: FAO, 2007.

[32] A. A. Ospina, Agroforestería. Aportes conceptuales, metodológicos y prácticos para el estudio agroforestal, Cali, Colombia: ACASOC. 2006.

[33] V. Palacios y J. C. Barrientos, "Importancia del huerto casero en la seguridad alimentaria. Caso de la comunidad indígena de camëntsá del Valle de Sibundoy, Colombia”, Ciencia Agro. Journal de Ciencias y Tecnología Agraria, vol. 2, n. ${ }^{\circ}$ 2, pp. 313-318, 2011.

[34] J. A. Perdomo, “Una propuesta metodológica para estimar los cambios sobre el valor de la propiedad: estudio de caso para Bogotá aplicando Propensity Score Matching y Precios Hedónicos Espaciales", Lecturas de Economía, vol. 73, pp. 49-65, 2010.

[35] A. M. Pérez, Identificación y potenciación de valores medioambientales para el desarrollo local y regional. Galicia: Universidad de Santiago de Compostela, 2013.

[36] G. L. Quintero, G. L. J. Rivera, y B. N. C. Marín, "Efecto de la distancia al acceso de trasporte masivo Megabús sobre el valor de la propiedad en la zona sub-centro del barrio Cuba", Grafías disciplinares de la UCP. vol. 19, pp. 73-78, 2012.

[37] F. D. A. Revollo, "Calidad de la vivienda a partir de la metodología de precios hedónicos para la ciudad de Bogotá - Colombia", Revista Digital Universitaria, vol. 10, n. ${ }^{\circ}$ 7, pp. 1-17, 2009.

[38] S. Rosen, "Hedonic Prices and Implicit Markets: Product Differentiation in Pure Competition", The Journal of Political Economy, vol. 82, n. ${ }^{\circ} 1$, pp. 34-55, 1974.

[39] N. Salazar, R. Steiner, A. Becerra, y J. Ramírez, "Los efectos del precio del suelo sobre el precio de la vivienda para Colombia", Ensayos sobre Política Económica, vol. 70, n. ${ }^{\circ}$ 31, pp. 17-66, 2013).

[40] J. D. Saphores y W. Li, "Estimating the value of urban Green areas: A hedonic pricing analysis of the single family housing market in Los Angeles, CA", Landscape and urban planning, vol. 3-4, n. ${ }^{\circ}$ 104, pp. 373-387, 2012.

[41] Secretaría del Medio Ambiente y Recursos Naturales (SEMARNAT). Valoración Económica de Servicios Ambientales Prestados por Ecosistemas: Humedales en México. México: INE.2001.

[42] E. Uribe, J. Mendieta, H. Rueda, y F. Carriazo, Introducción a la Valoración Ambiental, y estudios de caso. CEDE-COLCIENCIAS, Bogotá: Ediciones, Uniandes. 2003.

[43] F. Vásquez, J.C. Castilla, S. Gelcich, M.A. Quiroga, P. Carrasco, X. Paz y J. Riquelme Evaluación económica de los activos ambientales presentes en la red de reservas marinas decretadas en el país bajo la ley general de pesca y acuicultura, informe final de proyecto, Universidad de Concepción, Valparaíso, 2010.

[44] A. D. Vilamajó, C. M. Gispert, G. M. A Vales, E. A. González, y G. H. Rodríguez, "Los huertos familiares como reservorios de recursos fitogenéticos arbóreos y de patrimonio cultural en Rayón, México y el Volcán, Cuba", Etnobiología vol. 9, n. ${ }^{\circ}$ 1, 22-36, 2011.

[45] P. E. Viquez, "Caracterización del huerto mixto tropical La Asunción, Masatepe, Nicaragua". Agroforestería en las Américas (CATIE), vol. 1, n. ${ }^{\circ}$ 2, pp. 5-9, 1994.

[46] R. G. Walsh y J. B. Loomis, Recreation economic decisions: comparing benefits and costs. Pennsylvania: Venture Publishing Inc., 1986.

[47] Zambrano, M. M. "Formación de los precios de alquiler de viviendas en Machacala (Ecuador): análisis mediante el método de precios hedónicos", Spanish of Economics and Finance. vol. 39, n. ${ }^{\circ}$ 109, pp. 12-22, 2016.

[48] J.; Zhao, X. Cai y Z. Wang, (2013), "Comparing administered and market-based water allocation system sthrough a consisten tagent based modeling framework", Journal of Environmental Management, vol. 123, n. ${ }^{\circ} 1$, pp. 120-130, doi:10.1016/j.jenvman.2013.03.005 